\title{
Optical properties of asymmetric T-shaped quantum wires with large confinement
}

\author{
Langbein, Wolfgang Werner; Gislason, Hannes; Hvam, Jørn Märcher
}

Published in:

OSA Technical Digest Series, vol. 11

Publication date:

1997

Document Version

Publisher's PDF, also known as Version of record

Link back to DTU Orbit

Citation $(A P A)$ :

Langbein, W. W., Gislason, H., \& Hvam, J. M. (1997). Optical properties of asymmetric T-shaped quantum wires with large confinement. In OSA Technical Digest Series, vol. 11 (pp. 111-112). IEEE.

\section{General rights}

Copyright and moral rights for the publications made accessible in the public portal are retained by the authors and/or other copyright owners and it is a condition of accessing publications that users recognise and abide by the legal requirements associated with these rights.

- Users may download and print one copy of any publication from the public portal for the purpose of private study or research.

- You may not further distribute the material or use it for any profit-making activity or commercial gain

- You may freely distribute the URL identifying the publication in the public portal

If you believe that this document breaches copyright please contact us providing details, and we will remove access to the work immediately and investigate your claim. 


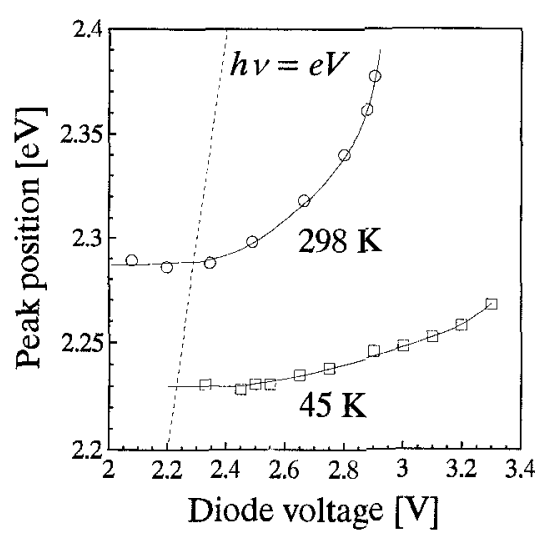

CTuO3 Fig. 2 Position of electroluminescence peak as a function of current measured at two different temperatures for LED \# 208. Note that $\mathrm{E}_{\mathrm{g}}(300 \mathrm{~K})=2.430 \mathrm{eV}$ and $\mathrm{E}_{\mathrm{g}}(45 \mathrm{~K})=2.505$ $\mathrm{eV}$.

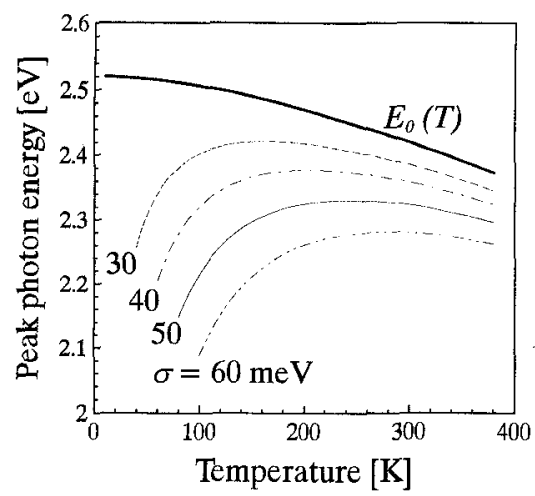

CTuO3 Fig. 3 Temperature shift of the emission peak calculated using the band tail model for $\mathrm{In}_{0.45} \mathrm{Ga}_{0.55} \mathrm{~N}$ with various values of the band tail width parameter $\sigma . \mathrm{E}_{0}(\mathrm{~T})$ temperature variation of band gap extrapolated from Ref. 6 .

bandgap energy of the electroluminescence peak and anomalous temperature shift) we developed a model in which we assume that the radiative transitions involve Gaussian-like tails of conduction and valence bands. The model can account for temperature dependence of light intensity and emission peak position, and for temperature insensitivity of emission bandwidth. The emission peak position can be expressed in this approach as:

$$
E(T)=E_{0}(T)-\frac{\sigma^{2}}{k T},
$$

where $E_{g}(T)$ is the distance between centers of Gaussian tails and $\sigma$ is an effective tail width. Figure 3 shows that we can obtain a positive temperature coefficient of the emission peak for large enough band tail width parameter $\sigma$.

Further work is required to determine the physical nature of band tail states involved in our model. The presence of band tails observed recently in $\mathrm{GaN}$ epi-layers ${ }^{4}$ can be related to the high density of dislocations (typically $10^{10}$ $\left.\mathrm{cm}^{-3}\right)^{5}$ and/or to large fluctuations of indium contents in the quantum well material.

Support from DARPA under Optoelec- tronic Materials Center is gratefully acknowledged.

${ }^{*}$ On leave from P. N. Lebedev Physics Institute, Moscow, Russia

${ }^{* *}$ On leave from High Pressure Research Center, Warsaw, Poland

${ }^{\dagger}$ On leave from Ecole Nationale Supérieure des Télécommunications, Paris, France

1. S. Nakamura, M. Senoh, N. Iwasa, S. Nagahama, T. Yamada, T. Mukai, Jpn. J. Appl. Phys. 34, L1335 (1995).

2. S. Nakamura, M. Senoh, S. Nagahama, N. Iwasa, T. Yamada, T. Matsushita, H. Kiyoku, Y. Sugimotu, Jpn. J. Appl. Phys. 35, L74 (1996).

3. S. Chichibu, T. Azuhata, T. Sota, S. Nakamura, presented at $38^{\text {th }}$ Electronic Materials Conference, June 1996, UC Santa Barbara, California.

4. C. H. Qiu, C. Hoggatt, W. Melton, M. W. Leksono, J. I. Pankove, Appl. Phys. Lett. 66, 2712 (1995).

5. S. D. Lester, F. A. Ponce, M. G. Craford, D. A. Steigerwald, Appl. Phys. Lett. 66, 1249 (1995)

6. K. Osamura, S. Naka, Y. Murakami, J. Appl. Phys. 46, 3432 (1975).

CTuO4 (Invited)

11:15 am

MOCVD growth and fabrication of groupIII nitrides for high-efficiency MQW LEDs

Steven P. DenBaars, P. Kozodoy, A. Abare, M. Mack, S. Keller, U. K. Mishra, L. A. Coldren, UC-Santa Barbara; S. Imagin, B. Kolbas, North Carolina State Univ.

MOCVD growth conditions have been found to have a critical influence on the performance of electronic and optoelectronic devices. Multiple quantum well (MQW) indium gallium nitride ( $\mathrm{InGaN}$ ) light-emitting diodes (LEDs) have also been grown and emit bright blue direct gap luminescence at a peak wavelength of $450 \mathrm{~nm}$. The luminescence peaked at $450 \mathrm{~nm}$ and the external quantum efficiency was $4.5 \%$ for the MQW structure at $20 \mathrm{~mA}$. High peak power output of $55 \mathrm{~mW}$ was measured under pulsed condition of $1 \mathrm{~A}$ for 3 microsec pulse width, which is amongst the highest reported pulsed power output for LED. Using a Ti:sapphire laser operating at $280 \mathrm{~nm}$ we have observed optically pumped lasting in In GaN with linewidth as narrow as $0.7 \mathrm{~nm}$. A sharp threshold at onset of stimulated emission and a strong nonlinear dependence of output emission on input power was observed.

\section{CTu05 (Invited)}

11:45 am

Fabrication and characterization of semiconductor quantum dot lasers

S. Charbonneau, Institute for Microstructural Sciences, National Research Council of Canada, Ottawa, Canada, KlA OR6; E-mail: sylvain.charbonneau@nrc.ca

In recent years, there has been a great deal of interest in the investigation of optical processes in zero-dimensional (0-D) heterostructures. The motivations that stimulate research in this field are related to the possibility of using these materials for studying basic quantum properties, as well as for their potential use in novel optoelectronic devices like quantum dot (QD) lasers. One fabrication method which has attracted considerable interest is the creation of self-assembled QDs during a morphological transition from two- to threedimensional growth, denominated StranskiKrastanow growth mode. In these structures, for which the typical QD diameter averages 20 $\mathrm{nm}$, the oscillator strength increases due to the extreme localization of the exciton wavefunction, and strong $0-D$ confinement effects are observed. Layers of quantum dots can be substituted for the normally used quantum wells in conventional edge emitting laser structures. The interest in QD lasers is based on several theoretical predicted advantages. Ideal QD lasers are expected to exhibit lower thresholds, higher differential gains, higher modulation speeds and less temperature sensitivity (high $\mathrm{T}_{\mathrm{o}}$ ).

The first part of this talk focuses on the intersublevel relaxation time of carriers in QDs estimated from time-resolved photoluminescence (PL) experiments. Although most QD structures do not display any significant excited-state emission in their PL emission spectrum, results have been obtained for slightly larger self-assembled QD (36 nm in diameter) in an $\mathrm{In}_{0.5} \mathrm{Ga}_{0.5} \mathrm{As} / \mathrm{GaAs}$ system, which displayed strong level-filling, and emission from the excited states (up to $\mathrm{n}=5$ ) for increasing excitation intensities. This nonequilibrium condition can be achieved from the filling of lower-confined states, thus forcing the carriers to remain in the excited state levels before relaxation can occur. Timeresolved PL experiments performed on that sample have been used to measure the ground state excitonic recombination time as well as the decay times for the excited states. From the measured lifetime of each energy level, under various excitation conditions, an estimate of the inter-sublevel relaxation time in the $\mathrm{QD}$ is obtained. These results show the influence of the small density of states of $0-\mathrm{D}$ systems on carrier dynamics.

In the second part of the talk, I describe the design, fabrication, and operating characteristics of red-emitting InAlAs $Q D$ laser grown on GaAs cladded by AlGaAs. The device exhibit a lasing wavelength of $\sim 700 \mathrm{~nm}$ at a temperature of $77 \mathrm{~K}$ with a lasing threshold current density of less than $100 \mathrm{~A} / \mathrm{cm}^{2}$.

CTu06

12:15 pm

Optical properties of asymmetric T-shaped quantum wires with large confinement

W. Langbein, H. Gislason, J. M. Hvam, Mikroelektronik Centret, The Technical University of Denmark, Building 345 east, DK-2800 Lyngby, Denmark

The fabrication of semiconductor nanostructures with an effective dimensionality lower than two is intensively pursued in view of the expected concentration of the density of states, enhancing the properties of optoelectronic devices. The quantum wires forming at the 


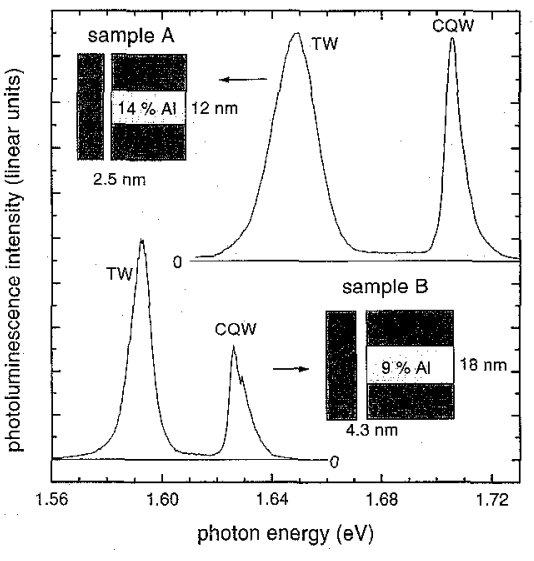

CTuO6 Fig. 1 PL of the T-wire samples A and $B$ at $40 \mathrm{~K}$ under nonresonant optical excitation. The structure parameters are given in the inserts.

T-shaped intersection of two quantum wells (QWs) ${ }^{1}$ have an advantage over quantum wires produced by lithographic methods or self-organized growth because the intersection can be realized by the cleaved edge overgrowth (CEO) technique ${ }^{2}$ with the high precision of the molecular beam epitaxy (MBE). These T-shaped structures show optically and electrically excited photoluminescence and lasing from the quantum wire state (TW state). ${ }^{3}$ To make them suitable for room-temperature applications, the confinement energy of the TW states (WCE) has to be increased to several $k_{\mathrm{B}} T$. We have maximized the WCE for given widths of the overgrown (110) GaAs QW (OQW) by a variation of the cleaved (001) QW (CQW). ${ }^{4}$ Increasing its width and aluminum content relative to a symmetric structure, while keeping its transition energy fixed at that of the OQW, the quantization energy of the TW state in the (001) direction is reduced and thus the WCE increased. The optimal width is determined by the onset of confinement of a second TW state, which is reducing the effective WCE to the separation between first and second state. We have determined this width by a numerical calculation of the electronic states using an isotropic effective mass model for the conduction band and a six-band $k \cdot p$ model for the valence band. The corresponding maximal WCE is doubled compared to symmetric $T$-shaped structures.

This prediction is confirmed by the properties of experimental structures (samples A, B) grown using the optimized parameters, which show WCEs of $56 \mathrm{meV}$ and $39 \mathrm{meV}$, respectively. The PL spectra of these samples are given in Fig. 1, together with the sample parameters.

The temperature dependence of the PL efficiency (Fig. 2) is dominated by the thermal activation of the carriers into the CQW. We find activation energies of $70 \mathrm{meV}$ and 32 $\mathrm{meV}$, for sample A and B, respectively. The influence of the thermal activation on the PL decay time $\tau$ can be modeled by $\tau^{-1}(T)=\tau_{\mathrm{r}}^{-1}$ $+\tau_{\text {esc }}^{-1} \exp \left(-E_{\mathrm{a}} / k_{\mathrm{B}} T\right)$, with the activation energy $E_{\mathrm{a}}$, the recombination time $\tau_{\mathrm{r}}$, and the escape time $\tau_{\text {esc }}$. A fit to the experimental data of sample B (inset of Fig. 2) results is $\tau_{\mathrm{r}}=330$ $\mathrm{ps}$ and $\tau_{\mathrm{esc}}=0.5 \mathrm{ps}$. The recombination time is comparable to the time measured for quantum

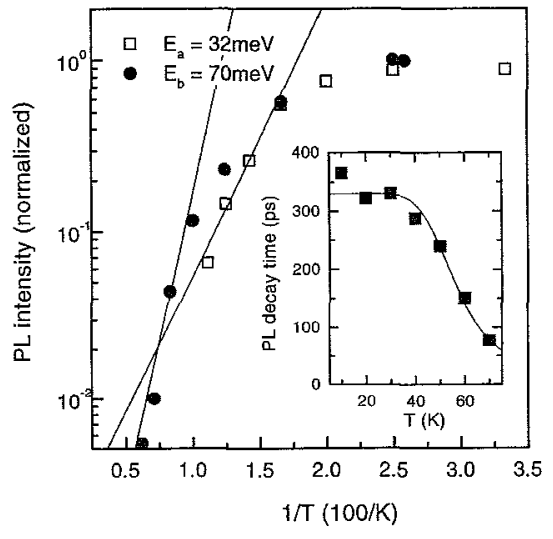

CTuO6 Fig. 2 Temperature dependence of the integrated PL intensity for sample A (full dots) and B (open squares), and of the PL decay time for sample $B$ after resonant picosecond excitation (inset) together with the fit curve.

wires on strained superlattices. ${ }^{5}$ The escape time of $0.5 \mathrm{ps}$ is reasonable for the involved phonon-absorption by the TW excitons. The time resolved TW PL spectra at low temperatures reveal the energy relaxation of the TW excitons within the localized wire states by phonon-assisted hopping.

1. Y-C. Chang, L. L. Chang, L. Esaki, Appl. Phys. Lett. 47, 1324 (1985).

2. L. Pfeiffer, et al., J. Crystal Growth 127, 849 (1993).

3. W. Wegscheider, et al., J. Crystal Growth 150, 285 (1995).

4. W. Langbein, H. Gislason, J. M. Hvam, Phys. Rev. B 54, (1996).

5. D. Gershoni, et al., Phys. Rev. B 50, R8930 (1994).

\section{CTuP \\ 1:00 pm-2:30 pm \\ Exhibit Hall}

CLEO Poster Session: 1

Wave Mixing and Photorefractives

\section{CTuP1}

Temperature dependence of electron mobility in $\mathrm{Bi}_{12} \mathrm{GeO}_{20}$ and $\mathrm{Bi}_{12} \mathrm{SiO}_{20}$ with use of the time-of-flight technique

D. Bloom, S. W. S. McKeever, Room 145 PS II, Department of Physics, Oklahoma State University, Stillwater, Oklahoma 74078-3072

The sillenites are technologically important materials because of their photorefractive properties. The photorefractive effect exhibited by these materials gives them potential for use in applications such as high density holographic storage, optical switching, optical filtering, and many others. Before commercial devices can be produced, however, the materials properties have to be understood. The mobility is an important parameter since it determines the maximum speed at which a device

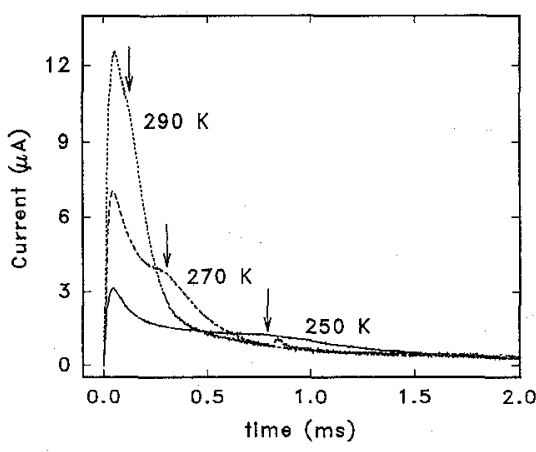

CTuP1 Fig. 1 Typical electron current transients measured in undoped $\mathrm{BGO}$ at the indicated temperatures. The arrows indicate the transit time of the fastest carriers.

made from the material can operate. Several measurements of mobility have been made in the sillenites, but the resulting values are spread over approximately 6 orders of magnitude. Values have been found from $\sim 5 \times 10^{-6}$ to $\sim 3 \mathrm{~cm}^{2} / V s$. This wide spread is probably due in part to impurity variations in crystals that were grown in different laboratories, but differing measuring techniques also play a role.

We measured drift mobilities in undoped and $0.3 \% \mathrm{Fe}$-doped $\mathrm{Bi}_{12} \mathrm{GeO}_{20}$ and undoped $\mathrm{Bi}_{12} \mathrm{SiO}_{20}$ between $\sim 210 \mathrm{~K}$ and $300 \mathrm{~K}$ using the time-of-flight (TOF) technique. In a TOF experiment $a$ thin sheet of free electrons and holes is generated by a strongly absorbed pulse of light, typically from a pulsed laser or a flash lamp. By applying a voltage to the sample either electrons or holes are driven into the bulk of the material depending on the polarity of the applied voltage. All of our current transients were produced by electrons. The hole current transients were too weak to detect. When the carriers reach the far electrode the current shows a "break" that is taken as the transit time of the fastest carriers. Typical transients measured in undoped BGO are shown in Fig. 1. From the time of the current break the drift mobility, $\mu_{d}$, of the fastest carriers can be calculated by use of

$$
\mu_{d}=\frac{L^{2}}{t_{T} V}
$$

where $L$ is the sample thickness, $t_{T}$ is the transit time, and $V$ is the applied voltage. We found that the mobility was independent of applied voltage and sample thickness.

We found that the shape of the current decay after the break was consistent with that expected for a Gaussian packet of carriers traveling through the sample. By fitting the transients we were able to determine the average carrier mobility and the width of the carrier packets. The fitting method will be described in the presentation. Fits to current transients measured in $0.3 \% \mathrm{Fe}$-doped $\mathrm{BGO}$ are shown in Fig. 2.

The temperature dependence of the average drift mobility is shown in Fig. 3. The values of the drift mobility range from $6.1 \times 10^{-4}$ to $0.10 \mathrm{~cm}^{2} / V_{s}$ in undoped $B G O$ and are about an order of magnitude smaller in both $0.3 \% \mathrm{Fe}$ doped BGO and undoped BSO. The temperature dependence is close to exponential indi- 